\title{
Hydrodynamic modes of a holographic $p$-wave superfluid
}

\author{
Raúl E. Arias ${ }^{a}$ and Ignacio Salazar Landea ${ }^{b}$ \\ ${ }^{a}$ Centro Atómico Bariloche, \\ 8400-S.C. de Bariloche, Río Negro, Argentina \\ ${ }^{b}$ IFLP-CONICET and Departamento de Fúsica, Facultad de Ciencias Exactas, \\ Universidad Nacional de La Plata, \\ CC 67, 1900, La Plata, Argentina \\ E-mail: rarias@cab.cnea.gov.ar, peznacho@gmail.com
}

ABSTRACT: In this work we analyze the hydrodynamics of a $p$ - wave superfluid on its strongly coupled regime by considering its holographic description. We obtain the poles of the retarded Green function through the computation of the quasi-normal modes of the dual AdS black hole background finding diffusive, pseudo-diffusive and sound modes. For the sound modes we compute the speed of sound and its attenuation as function of the temperature. For the diffusive and pseudo-diffusive modes we find that they acquire a non-zero real part at certain finite momentum.

KEYwords: Gauge-gravity correspondence, Spontaneous Symmetry Breaking, Black Holes, Holography and condensed matter physics (AdS/CMT)

ARXIV EPRINT: 1409.6357 


\section{Contents}

1 Introduction 1

2 Holographic $p$-wave superconductor 2

2.1 The model 3

2.2 Gauge field fluctuations 5

3 Quasinormal modes $\quad 6$

$\begin{array}{lr}3.1 \text { Longitudinal modes } & 6\end{array}$

$\begin{array}{lll}3.1 .1 & \text { Unbroken phase } & 7\end{array}$

$\begin{array}{lll}\text { 3.1.2 } & \text { Broken phase for } a_{x}, a_{t} \text { sector } & 7\end{array}$

$\begin{array}{ll}\text { 3.1.3 Broken phase for } a_{y} \text { sector } & 10\end{array}$

3.2 Transverse modes 11

$\begin{array}{lll}3.2 .1 & \text { Unbroken phase } & 11\end{array}$

$\begin{array}{lll}3.2 .2 & \text { Broken phase }- \text { sector I } & 12\end{array}$

$\begin{array}{lll}3.2 .3 & \text { Broken phase - sector II } & 13\end{array}$

4 Conclusions $\quad 14$

\section{Introduction}

The gauge/gravity correspondence [1-3] provides a useful tool to study quantum field theories in the strongly coupled regime. In the last years, this was applied to describe the behavior of condensed matter systems (see [4-7] for nice reviews). In the present work we will study the hydrodynamic modes of a $p$-wave superconductor using holography. In [8] the authors for a studied this system in QFT.

The order parameter for this class of superconductors is a vector field. Its gravity dual was proposed in the probe limit in [9] and the backreaction on the metric was analyzed in [10-12]. Holographically the $p$ - wave superconductor [9] can be modeled with an asymptotically AdS black hole with a $\mathrm{SU}(2)$ gauge field living on it. The boundary behavior of this gauge field gives the chemical potential and the order parameter of the superconductor. In particular, the chemical potential breaks the $\mathrm{SU}(2)$ gauge group to a $\mathrm{U}(1)$ subgroup and the condensate spontaneously breaks this $\mathrm{U}(1)$ subgroup and rotational symmetry. In this work we are going to take into account the probe limit approximation in which the gauge field doesn't deform the geometry of the space-time. Roughly speaking this approximation means that we have a small amount of charged (under the gauge group) degrees of freedom compared to the total number of degrees of freedom of the QFT.

The hydrodynamic theory studies the conservation equations for whatever symmetry the system has. In particular the conservation of the stress energy tensor and, if the system 
has some global symmetry, the conservation of the associated Noether current. The ansatz that solves these systems are called constitutive equations and because hydrodynamics is an effective theory they are functions of the derivatives of the temperature, the local velocity and the chemical potential. From those equations we can compute some transport properties like the diffusion constant, the first and second sound velocity, the charge conductivity, the charge density and the shear and bulk viscosities. Linear response theory allow us to know the dispersion relation for the hydrodynamics modes through the poles of the retarded Green function (see [13] for a nice review on relativistic hydrodynamics). Since we will work in the probe limit, we will only have information about the fluctuations of the modes associated to the Noether current. A more complete study of the system with backreaction could enlighten us about the stress energy tensor fluctuations.

As was shown in [14] the retarded Green function can be obtained holographically through the study of the quasinormal modes (QNM's) of the dual gravity background (see $[15,16]$ for reviews on the computation of QNM's in black hole geometries). Previous works on holographic hydrodynamics compute the hydrodynamic modes of $\mathcal{N}=4$ SYM $[17,18]$. The aim of the present work is to analyze the hydrodynamic modes of the $p$-wave superconductor through the study of the quasinormal modes spectrum of its holographic dual. In other words, we are going to study the poles of the current-current two point functions $\left\langle j_{i}^{a} j_{i}^{a}\right\rangle$ in the probe limit approximation. Here, the current $j_{x}^{1}$ is the order parameter of the $p$-wave superconductor. Moreover we are interested in the computation of the second sound of the superconductor. In the two fluid model of superconductivity the system is divided in two components, the normal fluid that contains elementary excitations like the phonon and roton and the superfluid that consists on the condensate. This model predicts the existence of a second sound mode which is the de-phased collective motion of the two fluids. We are going to use holography to compute this second sound in a $p$-wave superconductor (see [19, 20] for previous work on this direction). Moreover we are going to obtain diffusive and pseudo-diffusive modes.

In [21] the authors did a Gaussian quench in the order parameter of the gravity dual of a $s$-wave superfluid $[22,23]$. They showed that a universal late time behavior of the system is described by the poles of the retarded Green function that lie the closest to the real axis. Furthermore, it is discussed in [24] that the dynamical phase diagram can be extracted from the information of the QNM at equilibrium. Then, this is another application of our study of the quasinormal modes. They will describe the late time behavior of the quenched $p$-wave superconductor.

The program is the following. In section 2 we are going to review the gravity dual to the $p$-wave superconductor in the probe limit proposed on [9]. In section 3 we study the quasinormal spectrum and describe the second sound, the diffusive and pseudo-diffusive modes. Finally, the results are summarized in section 4 .

\section{Holographic $p$-wave superconductor}

In this section we are going to review the gravity dual found in [9] for a holographic $p$-wave superfluid. 
The order parameter in conventional superconductors comes from electron pairs that couple forming Cooper pairs. The state describing this pair must be symmetric and then only certain combinations are allowed. The $p$-wave superconductor is the name for those systems in which the relative orbital angular momentum between the electrons forming the Cooper pair is $l=1$. The superfluid ${ }^{3} \mathrm{He}-A$ is a real world example of a system with this order parameter.

We will work in the simplest set up and consider $\mathrm{SU}(2)$ as the gauge group. We will consider the system charged under the $\mathrm{U}(1)$ inside $\mathrm{SU}(2)$. This will explicitly break $\mathrm{SU}(2)$ down to $\mathrm{U}(1)$. The $p$-wave superconductor ansatz, breaks the remaining $\mathrm{U}(1)$ symmetry and the $\mathrm{SO}(2)$ symmetry associated to spatial rotations in the bidimensional boundary theory. The gravity solution that describes the strong coupling dynamics is as follows: a charged superconducting layer develops outside the horizon due to the interplay between the electric repulsion and the gravitational potential of the asymptotically AdS geometry. At high enough temperatures there is no hair outside the black hole and the solution is just a charged AdS black hole. Below a critical temperature $T_{c}$ a non-trivial gauge field with non-vanishing chemical potential on the boundary of the geometry and a sourceless nonvanishing condensate in the bulk appears, breaking the remaining U(1) gauge symmetry. An alternative formulation for a $p$-wave superfluid can be found in [25].

\subsection{The model}

The Einstein-Yang-Mills action fixed by the gauge symmetry reads

$$
S=\frac{1}{2 \kappa^{2}} \int d^{4} x\left(R-\frac{1}{4}\left(F_{\mu \nu}^{a}\right)^{2}+\frac{6}{L^{2}}\right),
$$

where $\kappa$ is the gravitational constant in four dimensions and $F_{\mu \nu}$ is the field strength of an $\mathrm{SU}(2)$ gauge field

$$
F_{\mu \nu}^{a}=\partial_{\mu} A_{\nu}^{a}-\partial_{\nu} A_{\mu}^{a}+g_{\mathrm{YM}} \epsilon^{a b c} A_{\mu}^{b} A_{\nu}^{c} .
$$

Here the index $a$ runs through the three $\mathrm{SU}(2)$ generators. We are going to work in the probe limit, that is large $\frac{g_{\mathrm{YM}}}{\kappa}$. By scaling the gauge field as $\tilde{A}=\frac{A}{g_{\mathrm{YM}}}$ we see that the large $g_{\mathrm{YM}}$ limit corresponds to the probe (non-backreacting) limit of the gauge field. Roughly speaking one can think that $\frac{1}{g_{\mathrm{YM}}^{2}}$ counts the degrees of freedom of the dual field theory that are charged under the $\mathrm{SU}(2)$ gauge group and $\frac{1}{\kappa_{(4)}^{2}}$ counts the total number of degrees of freedom. Then the probe limit means that we have a small number of charged degrees of freedom with respect to the total number. Moreover, taking this limit allow us to decouple the metric fluctuations from the gauge field fluctuations. This means that we are going to study the retarded Green functions for current-current expectation values, $G_{j j}^{R}$, and we are not going to catch up the transport coefficients that comes from the stress-energy tensor conservation. In this limit, the proposed background geometry reads

$$
d s^{2}=\frac{r^{2}}{L^{2}}\left(-f(r) d t^{2}+d x^{2}+d y^{2}\right)+\frac{L^{2}}{r^{2} f(r)} d r^{2},
$$

with $f(r)=1-\frac{r_{h}^{3}}{r^{3}}$ and $r_{h}$ standing for the location of the black hole horizon. From now on we set the scale $r_{h}=1$. The asymptotically AdS boundary is located at $r=\infty$ and the 
temperature of the horizon is $T_{h}=\frac{3}{4 \pi L^{2}}$. The ansatz for the gauge field has the form

$$
A=\phi(r) \tau^{3} d t+w(r) \tau^{1} d x .
$$

Here $A=A_{\mu}^{a} \tau^{a} d x^{\mu}$ with $\tau^{a}=\frac{\sigma^{a}}{2 i}$ and $\sigma^{a}$ the usual Pauli matrices. These $\mathrm{SU}(2)$ generators satisfy the standard algebra $\left[\tau^{a}, \tau^{b}\right]=\epsilon^{a b c} \tau^{c}$. A solution developing $w(r) \neq 0$ in the gauge field ansatz breaks the remaining $\mathrm{U}(1)$ gauge symmetry associated with rotations around $\tau^{3}$ (usually called $\left.\mathrm{U}(1)_{3}\right)$. We are looking for solutions that break this $\mathrm{U}(1)_{3}$ symmetry spontaneously and we are going to achieve this imposing a non trivial regular profile for the gauge field.

We will redefine our fields $\tilde{\phi}(r)=g_{\mathrm{YM}} L^{2} \phi(r), \tilde{w}(r)=g_{\mathrm{YM}} L^{2} w(r)$ in order to simplify the equations. This is equivalent to set $g_{\mathrm{YM}}=L=1$. The Maxwell equations $D_{\mu} F^{\mu \nu}=0$ on this geometry read

$$
\begin{array}{r}
\tilde{\phi}^{\prime \prime}(r)+\frac{2}{r} \tilde{\phi}^{\prime}(r)-\frac{\tilde{w}(r)^{2} \tilde{\phi}(r)}{r^{4} f(r)}=0, \\
\tilde{w}^{\prime \prime}(r)+\left(\frac{f^{\prime}(r)}{f(r)}+\frac{2}{r}\right) \tilde{w}^{\prime}(r)+\frac{\tilde{w}(r) \tilde{\phi}(r)^{2}}{r^{4} f(r)^{2}}=0 .
\end{array}
$$

The system (2.6) has the following behavior near the horizon

$$
\begin{aligned}
& \tilde{\phi}(r) \approx \phi_{1}^{h}(r-1), \quad r \rightarrow 1, \\
& \tilde{w}(r) \approx w_{0}^{h}+w_{2}^{h}(r-1)^{2}, \quad r \rightarrow 1 .
\end{aligned}
$$

On the other hand the near the boundary behavior for these equations reads

$$
\begin{aligned}
& \tilde{\phi}(r)=\mu+\frac{\rho}{r}, \quad r \rightarrow \infty, \\
& \tilde{w}(r)=\frac{<j_{x}^{1}>}{r}, \quad r \rightarrow \infty .
\end{aligned}
$$

where $\mu$ is the chemical potential and $\rho$ the charge density of the dual field theory. The expectation value of the current $\left\langle j_{x}^{1}\right\rangle$ is the order parameter of the superfluid phase.

We will express our results in the grand canonical ensemble, i.e. at fixed chemical potential. Then the physical temperature of the system will be determined by the following re-scaled dimensionless magnitude $T=T_{h} / \mu$.

Note that in order to have a gravity dual of a spontaneously broken symmetry we need to have the leading term $w_{0}^{b} r^{0}=0$ in the boundary behavior of $\tilde{w}(r)$ because this is, according to the AdS/CFT dictionary, the source for $\left\langle j_{x}^{1}\right\rangle$. The solution is found using a shooting technique and the desired solution is obtained for values of the horizon coefficients $\phi_{1}^{h}, w_{0}^{h}$ that satisfy $w_{0}^{b}\left(\phi_{1}^{h}, w_{0}^{h}\right)=0$.

Exploring the space of parameters we find that a solution with $w(r) \neq 0$ only exist for low enough temperatures, which translates into a phase transition from a normal to a broken phase characterized by the order parameter $\left\langle j_{x}^{1}\right\rangle$, as shown in figure 1 .

The free energy for this solution was computed in [9], certifying that the broken phase is indeed thermodynamically preferred. When going to lower temperatures than those shown in figure 1 the probe limit breaks down and backreaction on the metric must be considered [10-12]. For analytical computations in this system see [26]

In what follows of this section we are going to consider fluctuations of the gauge field. 


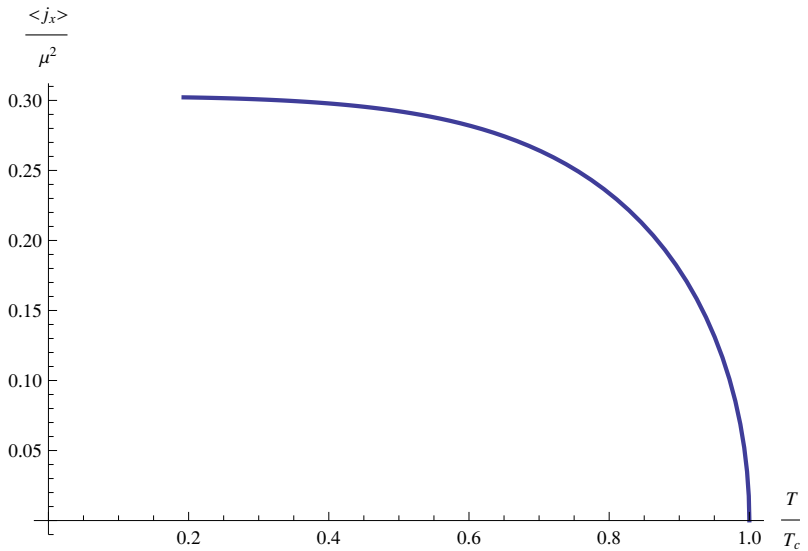

Figure 1. Re-scaled order parameter $\left\langle j_{x}^{1}>/ \mu^{2}\right.$ as a function of the temperature showing a second order phase transition for low enough temperatures.

\subsection{Gauge field fluctuations}

In order to study the QNM spectrum of this background we are going to consider the following fluctuations of the $\mathrm{SU}(2)$ gauge field

$$
\begin{aligned}
\mathcal{A}= & \left(\phi(r) \tau^{3}+\lambda \sum_{i=1}^{3} \delta A_{t}^{i}(t, x, y, r) \tau^{i}\right) d t+\left(w(r) \tau^{1}+\lambda \sum_{i=1}^{3} \delta A_{x}^{i}(t, x, y, r) \tau^{i}\right) d x \\
& +\lambda \sum_{i=1}^{3} \delta A_{y}^{i}(t, x, y, r) \tau^{i} d y
\end{aligned}
$$

where we fix the gauge $a_{r}^{i}=0$ and $\lambda$ is the expansion coefficient. Since we are interested in the QNM spectrum we will work at linear order in $\lambda$.

To have simpler equations of motion for this fluctuations we can divide them in different modes. In particular we are going to study modes that propagate in a longitudinal or transverse direction with respect to the direction of the condensate. ${ }^{1}$

\section{Longitudinal modes.}

$$
\delta A_{t, x, y}^{i}(t, x, y, r)=a_{t, x, y}^{i}(r) e^{-i \omega t+i k_{x} x}
$$

Transverse modes.

$$
\delta A_{t, x, y}^{i}(t, x, y, r)=a_{t, x, y}^{i}(r) e^{-i \omega t+i k_{y} y},
$$

Analyzing the modes in an arbitrary direction in the fashion of $[27,28]$ would be interesting but would require a bigger computational power.

In the next section we are going to study the solution to the equations of motion for the fluctuations $a_{t, x, y}^{i}(r)$ imposing ingoing boundary conditions at the horizon of the black

\footnotetext{
${ }^{1}$ Note that both sectors should coincide in the zero momentum limit and differences should arise only when considering $k \neq 0$.
} 
hole $^{2}$ of the form

$$
\begin{aligned}
a_{x, y}^{i} & \approx a_{x_{h}, y_{h}}^{i}(r-1)^{-\frac{i \omega}{3}}+\cdots, \\
a_{t}^{i} & \approx a_{t_{h}}^{i}(r-1)^{-\frac{i \omega}{3}+1}+\cdots .
\end{aligned}
$$

At the boundary we are going to impose that the leading behavior vanishes. This requirement will quantize the frequency, giving us the quasinormal spectrum of the system.

These quasinormal frequencies are the poles of the retarded Green function in the dual field theory. Furthermore, we are going to study the small momentum regime of these modes, which will give us the hydrodynamic regime of the $p$-wave superfluid.

Generically the equations of motion for the perturbations will appear coupled in sectors. To deal with this issue we will use the determinant method developed in [20] in order to solve the system. The desired quasinormal frequencies $\omega\left(k_{i}\right)$ are obtained from the roots of the determinant of the matrix formed with solutions to the equations evaluated at the boundary. In order to get an squared matrix, we will need an independent solution for each coupled equation.

Typically the number of linearized equations of motion for the fluctuations is greater than the number of free horizon parameters. This is due to constraint equations that relate some near horizon parameters. When this happens we will take advantage of the existence of (algebraic) pure gauge solutions in order to get as many independent solutions as equations of motion.

\section{Quasinormal modes}

In this section we are going to find the spectrum of exitations of the $p$-wave superconductor in the hydrodynamic limit. The analysis of these hydrodynamics modes can be done studying the QNM frequencies of the gravity dual. Here we are going to study the QNM spectrum of the geometry reviewed in section 2 and we will numerically solve the equations of motion for the fluctuations (2.10) and (2.11). In particular we are going to study its solutions in the unbroken (normal) and broken (superfluid) phase. As a first step we will study the normal phase which is going to give us information about the number of modes that we have. In a second step we will follow this modes in the superconducting phase and study their behavior.

\subsection{Longitudinal modes}

In this subsection we are going to study the following fluctuations of the gauge field:

$$
\begin{aligned}
& \delta A_{x}^{j}(t, r, x)=a_{x}^{j}(r) e^{i k_{x} x-i \omega t}, \\
& \delta A_{y}^{j}(t, r, x)=a_{y}^{j}(r) e^{i k_{x} x-i \omega t} \\
& \delta A_{t}^{j}(t, r, x)=a_{t}^{j}(r) e^{i k_{x} x-i \omega t}
\end{aligned}
$$

where $j$ runs over the three $\mathrm{SU}(2)$ index. As we shall see the equations of motion for the $\delta A_{y}^{j}$ fluctuation decouples and can be analyzed separately.

\footnotetext{
${ }^{2}$ This ensures that we are dealing with retarded Green functions. On the other hand, outgoingness at the horizon corresponds to the holographic computation of the advanced Green functions.
} 


\subsubsection{Unbroken phase}

In the normal phase, where $w(r)=0$, the 6 equations of motion for the fluctuations (3.1) and (3.3) decouple in 2 (background independent) equations for $a_{t}^{3}, a_{x}^{3}$ and 4 equations coupling $a_{x}^{1}, a_{x}^{2}, a_{t}^{1}, a_{t}^{2}$.

The sector with color indices $1-2$ gives us the mode that will drive the instability towards the superfluid phase. Following this mode through the phase transition, it will become the Goldstone mode of the broken phase.

On the other hand, the sector with color index 3, gives the diffusive mode of the normal phase with a dispersion relation $\omega=-i k^{2}$ [29]. Going further from the origin in the $\omega$ complex plane, the quasinormal frequencies at zero momentum are know analytically to be [29] $\omega=-i \frac{3}{2} n$, with $n$ being a positive integer number. The fate of these modes in the broken phase will be address in the following sections.

The behavior in the near horizon limit of the four equations of the $1-2$ sector allows to fix two of the horizon parameters in term of the others. In order to have a solution for this system we must use two pure gauge solutions (see eq. (3.5)). Instead, for the 3 -sector we need just one pure gauge solution $a_{t}^{3}=-\omega, a_{x}^{3}=k$ in order to determine the system.

On the other hand, the 3 equations for the fluctuations in the $y$ component (3.2) are separated in one equation for $a_{y}^{3}$ (which is temperature independent) and two coupled equations for $a_{y}^{1}, a_{y}^{2}$. In this case there is no reason to search for pure gauge solutions because the system is well defined. The equation for $a_{y}^{3}$ was previously studied in [29] and was shown that there is no a solution satisfying the Dirichlet condition at the AdS boundary compatible with the hydrodynamic approximation. Then, these set of equations give two modes coming from the $1-2$ sector. One of them is going to give a diffusive mode in the broken phase and the remaining mode is going to be a pseudo-diffusive.

Note that at zero momentum there is no distinction between $x$ and $y$ sectors since the rotational symmetry is not broken yet.

\subsubsection{Broken phase for $a_{x}, a_{t}$ sector}

Now, with a non vanishing condensate we must solve the following system with 6 equations and 6 unknowns

$$
\begin{aligned}
a_{t}^{3 \prime \prime}+\frac{-w\left(2 i k_{x} a_{t}^{2}+2 a_{x}^{1} \phi+i \omega a_{x}^{2}\right)-2 r^{3} f a_{t}^{3 \prime}+a_{t}^{3}\left(w^{2}+k_{x}^{2}\right)+k_{x} \omega a_{x}^{3}}{r^{4} f} & =0, \\
a_{t}^{2 \prime \prime}-\frac{\left.-2 r^{3} f a_{t}^{2 \prime}+a_{t}^{2} w^{2}+k_{x}^{2} a_{t}^{2}-i\left(2 k_{x} a_{t}^{3} w+k_{x} a_{x}^{1} \phi\right)+\omega a_{x}^{3} w\right)+k_{x} \omega a_{x}^{2}}{r^{4} f} & =0, \\
a_{x}^{3 \prime \prime}-\frac{a_{t}^{1} w \phi-\omega\left(i a_{t}^{2} w+k_{x} a_{t}^{3}+\omega a_{x}^{3}\right)-f r^{3} a_{x}^{3 \prime}\left(r f^{\prime}+2 f\right)}{r^{4} f^{2}} & =0, \\
a_{x}^{1 \prime \prime}+\frac{k_{x} \omega a_{t}^{1}+\phi\left(i k_{x} a_{t}^{2}+2 a_{t}^{3} w+2 i \omega a_{x}^{2}\right)+r^{3} f a_{x}^{1 \prime}\left(r f^{\prime}+2 f\right)+a_{x}^{1}\left(\phi^{2}+\omega^{2}\right)}{r^{4} f^{2}} & =0 \\
a_{x}^{2 \prime \prime}+\frac{-i\left(\phi\left(k_{x} a_{t}^{1}+2 \omega a_{x}^{1}\right)+\omega a_{t}^{3} w\right)+k_{x} \omega a_{t}^{2}+r^{3} f a_{x}^{2 \prime}\left(r f^{\prime}+2 f\right)+a_{x}^{2}\left(\phi^{2}+\omega^{2}\right)}{r^{4} f^{2}} & =0, \\
a_{t}^{1 \prime \prime}-\frac{k_{x}^{2} a_{t}^{1}-2 r^{3} f a_{t}^{1 \prime}+k_{x} \omega a_{x}^{1}+\phi\left(-a_{x}^{3} w+i k_{x} a_{x}^{2}\right)}{r^{4} f} & =0,
\end{aligned}
$$


where prime means derivative with respect to the radial coordinate $r$. The near horizon behavior of the equations allow us to fix 3 of the IR parameters. Then we need to use 3 pure gauge solutions in order to have a well posed system of equations. The pure gauge solutions are parametrized by $\lambda_{i}$ and read

$$
\begin{array}{llrl}
a_{x}^{1}=-\lambda_{2} k_{x}, & a_{x}^{2}=i \lambda_{1} w(r), & a_{x}^{3}=-\lambda_{1} k_{x}-i \lambda_{3} w(r), \\
a_{t}^{1}=\lambda_{2} \omega+i \lambda_{3} \phi(r), & a_{t}^{2}=-i \lambda_{2} \phi(r)+\lambda_{3} \omega, & a_{t}^{3}=\lambda_{1} \omega .
\end{array}
$$

We found two kind of hydrodynamic modes in this sector: two sound modes and a diffusive mode.

Sound modes. We have two sound modes that satisfy the dispersion relation

$$
\omega\left(k_{x}\right)= \pm v_{x} k_{x}-i \Gamma_{x} k_{x}^{2} .
$$

In a superfluid the ordinary sound is due to fluctuations of the density. The hydrodynamic equations for the two fluid model predicts the existence of a different sound produced by temperature or entropy fluctuations. This sound is called second sound and it depends strongly on the temperature. Along this work the second sound velocity is going to be denoted with $v_{i}$, with $i$ being the spatial direction index. The function $\Gamma_{x}(T)$ gives the imaginary part of the dispersion relations (3.6). It is called second sound attenuation, and is related with the mean free path of the quasiparticles.

In figure 2 we plot the second sound velocity as function of the temperature. Since we have a second order phase transition we expect everything to match at one and the other side of the critical point. The notion of second sound only makes sense in the two component fluid model of superconductivity, then is expected the vanishing sound velocity at $T=T_{c}$ where just normal fluid remains and there is no superfluid component. Furthermore, in the normal phase this QNM becomes massive.

Another interesting feature of figure 2 is the change in the slope for $T / T_{c} \sim 0.45$. This is similar to the results obtained in experiments with ${ }^{4} \mathrm{He}$ in $[30,31]$ and theoretically using a variational approach in [32].

In the right panel of figure 2 we can see the temperature dependence of the second sound attenuation. Note that there is a non vanishing attenuation at the critical temperature $\left(\Gamma_{s}=0.273131 T_{c}\right.$ for $\left.T=0.9992 T_{c}\right)$ and it vanishes for very low temperatures. Similar behavior for the attenuation near the critical temperature was obtained in [33] for the normal sound of an $\mathcal{N}=2$ plasma and in [20] for the second sound in the gravity dual of an $s$-wave superconductor.

Diffusive modes. These modes satisfy the following relation

$$
\omega\left(k_{x}\right)=-i D_{x} k_{x}^{2}
$$

with $D_{x}$ the diffusive constant. The diffusion modes are expected in a two fluid model of superfluidity because they are naturally related with the normal fluid component. As was mentioned before in the discussion of the unbroken phase this diffusive mode comes from the $a_{x}^{3}, a_{t}^{3}$ sector of the equations of motion. 

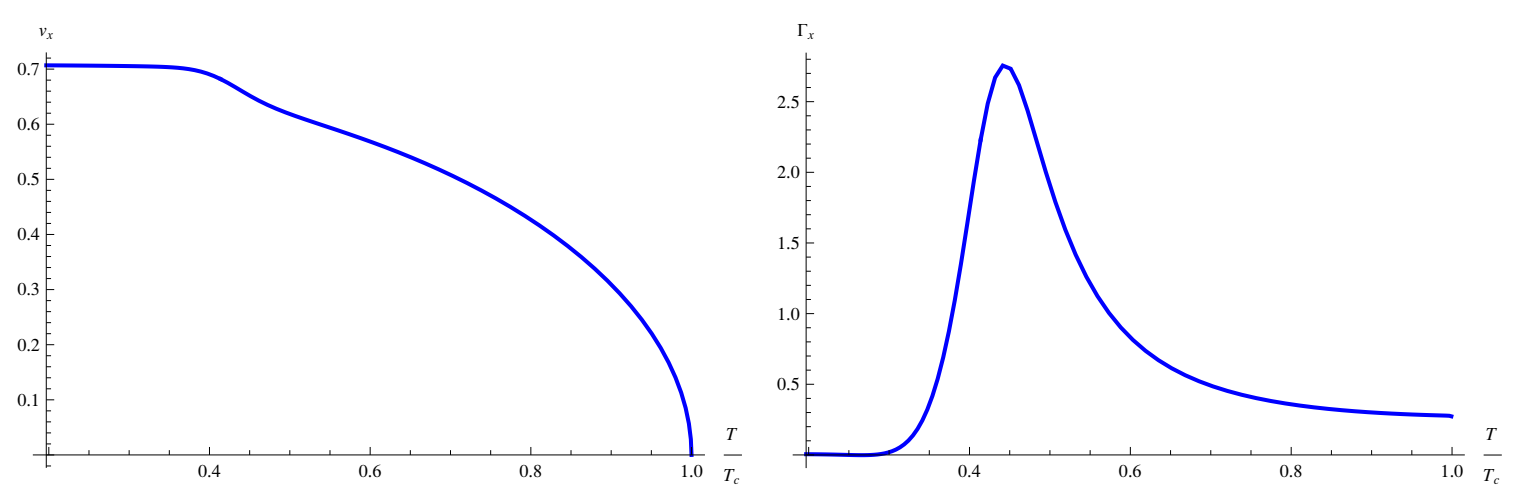

Figure 2. Second sound velocity (left) and attenuation coefficient (right) as a function of the temperature. The sound velocity vanishes (as expected) at the critical temperature.
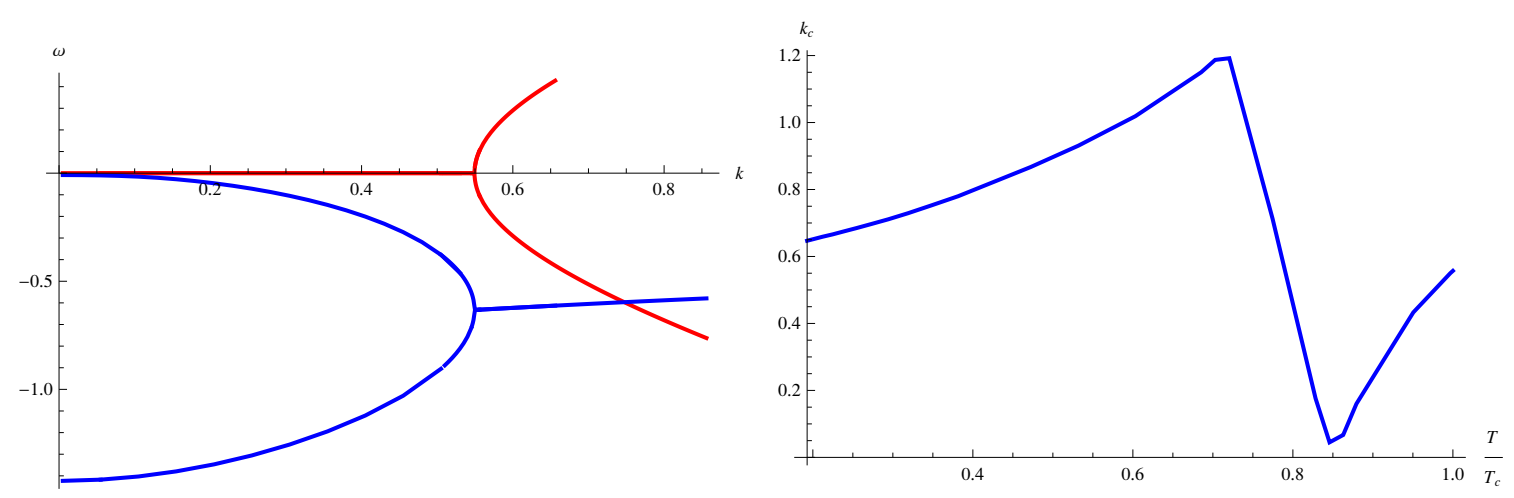

Figure 3. (Left) Spectrum for the diffusive mode. The blue curves correspond to the imaginary part of $\omega(k)$ while the red curves to its real part. The lowest lying of the blue curves corresponds to the first excited mode. We observe that at some value of $k=k_{c}$ it meets the hydrodynamic mode and they acquire a non-vanishing real part. (Right) Value of the momentum for which the diffusive and first excited mode meets as a function of the temperature.

Figure 3 shows the dispersion relation for the diffusive modes at $T=0.77458 T_{c}$. The red lines are the real part of $\omega$ and the blue lines its imaginary part. The branch with the lowest imaginary part corresponds to the first excited mode. Note that at some critical value of $k=k_{c}$ it meets the diffusive mode and they both acquire a real part. This critical value of $k$ is temperature dependent and its non vanishing at $T=T_{c}$. This is because the blue "bubble" is made of the hydrodynamic mode and the first excited mode. We will see that $k_{c}(T)$ has a different behavior for the diffusive modes in the transverse fluctuations. Interestingly in [34-37] this kind of "bubble" behavior for the hydrodynamic diffusive mode was observed for geometries originated from different brane configurations.

It is interesting to discuss the existence of this $k_{c}$ considering the discussion in [21, 24]. Since we expect these modes to rule the late time behavior of a quench, we can interpret this result as follows: during an inhomogeneous quench with a characteristic wavelength $k$, the order parameter will have an oscillatory or purely decaying behavior depending on weather $k$ is larger or smaller than $k_{c}$ at the final temperature of the quench. 

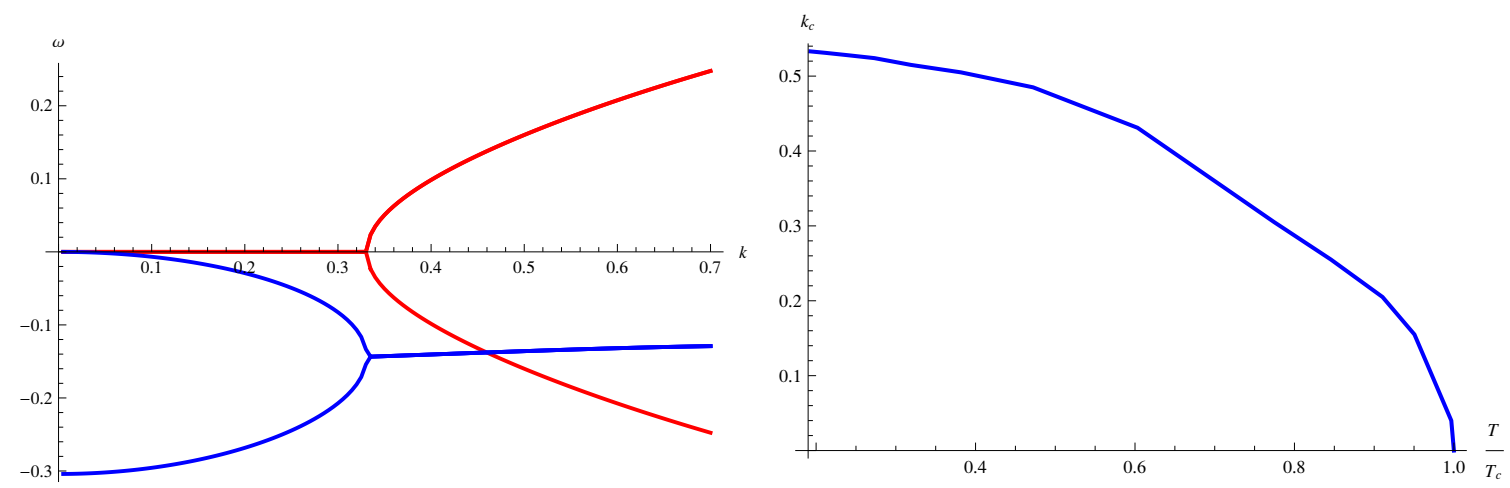

Figure 4. (Left) Diffusive and pseudo-diffusive mode for the $a_{y}$ fluctuations. The blue and red curves correspond to the imaginary and real part of the dispersion relation. (Right) Dependence on the temperature of the critical value $k_{c}$ where the two poles meet and acquire a non-vanishing real part.

\subsubsection{Broken phase for $a_{y}$ sector}

The equations of motion for the the $a_{y}^{i}$ fluctuations decouple from the $a_{x}^{i}$ perturbations previously analyzed and reads

$$
\begin{aligned}
a_{y}^{3 \prime \prime}-\frac{a_{y}^{3}\left(f\left(w^{2}+k_{x}^{2}\right)-\omega^{2}\right)-f\left(r^{3} a_{y}^{3 \prime}\left(r f^{\prime}+2 f\right)-2 i k_{x} a_{y}^{2} w\right)}{r^{4} f^{2}} & =0, \\
a_{y}^{2 \prime \prime}+\frac{-2 i \omega a_{y}^{1} \phi-f r^{3} a_{y}^{2 \prime}\left(r f^{\prime}+2 f\right)+a_{y}^{2}\left(f\left(w^{2}+k_{x}^{2}\right)-\phi^{2}-\omega^{2}\right)-2 i k_{x} a_{y}^{3} f w}{r^{4} f^{2}} & =0 .
\end{aligned}
$$

The lowest lying solutions are a pseudo-diffusive mode of the form

$$
\omega\left(k_{x}\right)=-i \tilde{D}_{x} k_{x}^{2}-i \gamma(T)
$$

and a proper diffusive mode with $\gamma=0$. Here $\tilde{D}_{x}$ is the diffusive constant and $\gamma$ is a real parameter that shifts the pole from its unbroken phase position. Then, at zero momentum we have a non-vanishing $\omega\left(k_{x}\right)$.

A typical solution is shown in figure 4 for $T=0.7745 T_{c}$ but its qualitative behavior is independent of the temperature. The blue curves correspond to the imaginary part of $\omega(k)$ while the red ones correspond to the real part of $\omega(k)$. The diffusive mode comes from the $a_{y}^{3}$ fluctuation in the unbroken phase and the coupled equations for $a_{y}^{1,2}$ gives the lower blue branch of the plot. Note the similitude with the diffusive mode for the longitudinal fluctuation shown in figure 3. This similitude is just apparent because in the present case the two blue branches correspond to a diffusive and a pseudo-diffusive mode. In figure 3 instead, the branches correspond with a diffusive mode and the firs exited mode. Again we have a critical value for the momentum $k$ where the modes meet and acquire non zero real part. Figure 4 shows how this critical value $k_{c}$ depends on the temperature.

The fact that the diffusive mode acquires a mass in the broken phase was previously observed in [20] in the context of holographic $s$-wave superfluids. On the other hand, the 
coupling between different (pseudo)diffusive modes, allows them to acquire a real part in their mass or dispersion relation. A similar behavior was observed in [24] in the context of $s$-wave U(2) superfluids.

\subsection{Transverse modes}

In this section we are going to study the fluctuations that propagate in the transversal direction with respect to the condensate, i.e. those given by equation (2.11). Due to the rotational symmetry of the unbroken phase we expect the same number of hydrodynamic modes that in the longitudinal case.

In this case we have two sets of fluctuations that can be consistently solved. The first one (which we are going to call sector $I$ ) reads

$$
\begin{aligned}
& \delta A_{x}^{j}(t, r, y)=a_{x}^{j}(r) e^{i k_{y} y-i \omega t}, \\
& \delta A_{y}^{3}(t, r, y)=a_{y}^{3}(r) e^{i k_{y} y-i \omega t}, \\
& \delta A_{t}^{3}(t, r, y)=a_{t}^{3}(r) e^{i k_{y} y-i \omega t},
\end{aligned}
$$

with $j=1,2$. From this sector we will find two sound modes and one diffusive mode.

The second consistent fluctuations (called sector II from now on) read

$$
\begin{aligned}
& \delta A_{x}^{3}(t, r, y)=a_{x}^{3}(r) e^{i k_{y} y-i \omega t}, \\
& \delta A_{y}^{j}(t, r, y)=a_{y}^{j}(r) e^{i k_{y} y-i \omega t}, \\
& \delta A_{t}^{j}(t, r, y)=a_{t}^{j}(r) e^{i k_{y} y-i \omega t},
\end{aligned}
$$

with $j=1,2$ and they lead to one diffusive and one pseudo-diffusive modes.

\subsubsection{Unbroken phase}

Since we defined our transverse or parallel modes according to the direction of the momentum with respect to the condensate, the transverse and parallel modes coincide in the normal phase. Nevertheless we will review them again here and discuss them according on how they couple in the broken phase. This is important since the continuity of the QNM across the phase transition will be one of our checks to the results obtained numerically.

For sector $I$ we have two sets of equations. One of them couples the fluctuations $a_{t}^{3}, a_{y}^{3}$ and are temperature independent. In [29] was shown that they give a diffusive mode. The remaining two equations couples $a_{x}^{1}, a_{x}^{2}$ and we found that they give the mode that drives the instability and evolves into the Goldstone mode when $T<T_{c}$. This Goldstone mode is of course massless and will give us at fine momentum a sound velocity. Thats why we will call it also sound mode.

For the sector $I I$ we have one decoupled equation for the $a_{x}^{3}$ fluctuation and four coupled equations for the remaining fluctuations. The decoupled equation was previously studied in [29] and was shown that it does not give a solution satisfying the boundary conditions compatible with the hydrodynamic limit, but just massive modes. The remaining equations are going to give two modes in the broken phase, one diffusive and one pseudo-diffusive mode. 

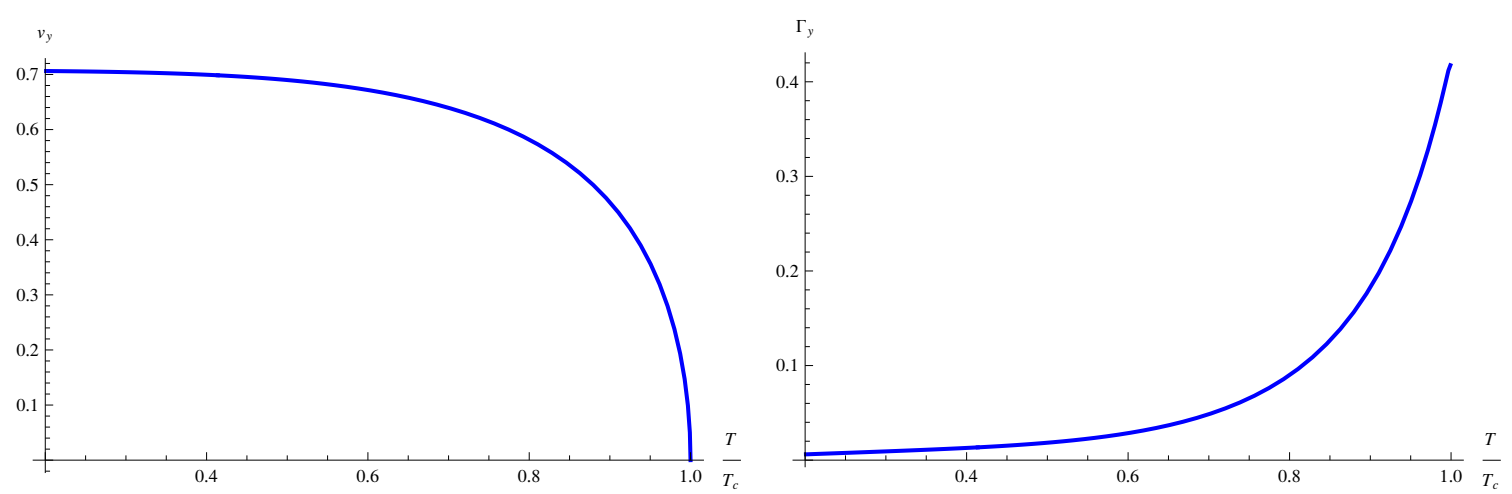

Figure 5. Second sound velocity (left) and attenuation coefficient (right) in the transversal direction as a function of the temperature. The sound velocity vanishes (as expected) at the critical temperature.

\subsubsection{Broken phase - sector I}

The equations of motion related to this sector read

$$
\begin{aligned}
a_{y}^{3 \prime \prime}-\frac{-k_{y} w a_{t}^{3}-f\left(r^{3} a_{y}^{3 \prime}\left(r f^{\prime}+2 f\right)+i k a_{x}^{2} w\right)+a_{y}^{3}\left(f w^{2}-\omega^{2}\right)}{r^{4} f^{2}} & =0, \\
a_{t}^{3 \prime \prime}-\frac{-2 r^{3} f a_{t}^{3 \prime}+a_{t}^{3}\left(w^{2}+k_{y}^{2}\right)+w\left(2 g a_{x}^{1} \phi+i \omega a_{x}^{2}\right)+k_{y} \omega a_{y}^{3}}{r^{4} f} & =0, \\
a_{x}^{1 \prime \prime}+\frac{2 \phi\left(a_{t}^{3} w+i \omega a_{x}^{2}\right)+r^{3} f a_{x}^{1 \prime}\left(r f^{\prime}+2 f\right)+a_{x}^{1}\left(\phi^{2}-f k_{y}^{2}+\omega^{2}\right)}{r^{4} f^{2}} & =0, \\
a_{x}^{2 \prime \prime}-\frac{-a_{x}^{2}\left(\phi^{2}-f k_{y}^{2}+\omega^{2}\right)+i\left(\omega a_{t}^{3} w+2 \omega a_{x}^{1} \phi+i r^{3} f a_{x}^{2 \prime}\left(r f^{\prime}+2 f\right)+k_{y} a_{y}^{3} f w\right)}{r^{4} f^{2}} & =0 .
\end{aligned}
$$

We again need to find a pure gauge solution

$$
a_{x}^{1}=0, \quad a_{x}^{2}=-i w(r), \quad a_{y}^{3}=-k_{y}, \quad a_{t}^{3}=\omega
$$

in order to use the determinant method. Then, we find from (3.16) two sound modes and one diffusive mode.

Sound modes. We have two sound modes that satisfy the dispersion relation

$$
\omega\left(k_{y}\right)= \pm v_{s} k_{y}-i \Gamma_{y} k_{y}^{2},
$$

in the hydrodynamic limit

In figure 5 we plot the second sound velocity as a function of the temperature. Again, we observe the expected vanishing behavior at $T=T_{c}$. On the other hand, in the low temperature limit we see that it has the same value that in the longitudinal case. In figure 5 we observe the transverse second sound attenuation as a function of the temperature. Note that as in the longitudinal case it tend to zero in the low temperature limit and takes a finite value at the critical temperature $\left(\Gamma_{y}=0.4175 T_{c}\right.$ at $\left.T=0.9992 T_{c}\right)$. This behavior is analog to what was found in [20] for the $s$-wave case. 
Diffusive mode. The low momentum dispersion relation for a diffusive mode reads

$$
\omega\left(k_{y}\right)=-i D_{y} k_{y}^{2}
$$

and the qualitative behavior is analog to that in figure 3 where the lowest blue line is the imaginary part of the first excited mode and the upper blue line is the pseudo-diffusive mode. Again, they meet at some critical value $k_{c}$ and its qualitative behavior is similar to the one shown in figure 3.

\subsubsection{Broken phase - sector II}

The equations of motion for this sector read

$$
\begin{aligned}
a_{x}^{3 \prime \prime}+\frac{-a_{t}^{1} w \phi+i \omega a_{t}^{2} w+r^{4} f a_{x}^{3 \prime} f^{\prime}+2 r^{3} f^{2} a_{x}^{3 \prime}+a_{x}^{3}\left(\omega^{2}-k_{y}^{2} f\right)+i k_{y} a_{y}^{2} f w}{r^{4} f^{2}} & =0, \\
a_{y}^{1 \prime \prime}+\frac{k_{y} \omega a_{t}^{1}+i \phi\left(k_{y} a_{t}^{2}+2 \omega a_{y}^{2}\right)+r^{3} f a_{y}^{1 \prime}\left(r f^{\prime}+2 f\right)+a_{y}^{1}\left(\phi^{2}+\omega^{2}\right)}{r^{4} f^{2}} & =0, \\
a_{y}^{2 \prime \prime}+\frac{-i \phi\left(k_{y} a_{t}^{1}+2 \omega a_{y}^{1}\right)+k_{y} \omega a_{t}^{2}-i k_{y} a_{x}^{3} f w+r^{3} f a_{y}^{2 \prime}\left(r f^{\prime}+2 f\right)+a_{y}^{2}\left(\left(\phi^{2}-f w^{2}\right)+\omega^{2}\right)}{r^{4} f^{2}} & =0, \\
a_{t}^{1 \prime \prime}-\frac{-2 r^{3} f a_{t}^{1 \prime}+k_{y}^{2} a_{t}^{1}+\phi\left(-a_{x}^{3} w+i k_{y} a_{y}^{2}\right)+k_{y} \omega a_{y}^{1}}{r^{4} f} & =0, \\
a_{t}^{2 \prime \prime}-\frac{-2 r^{3} f a_{t}^{2 \prime}+a_{t}^{2}\left(w^{2}+k_{y}^{2}\right)-i\left(\omega a_{x}^{3} w+k_{y} a_{y}^{1} \phi\right)+k_{y} \omega a_{y}^{2}}{r^{4} f} & =0 .
\end{aligned}
$$

Solving this equations of motions and using the pure gauge solutions

$$
a_{x}^{3}=0, \quad a_{y}^{1}=-\lambda_{1} k_{y}, \quad a_{y}^{2}=-\lambda_{2} k_{y}, \quad a_{t}^{1}=\lambda_{1} \omega+i \lambda_{2} \phi(r), \quad a_{t}^{2}=-i \lambda_{1} \phi(r)+\lambda_{2}(3.21)
$$

we find a pseudo-diffusive and a diffusive mode in the hydrodynamic regime.

Pseudo-diffusive mode. As in the longitudinal case, we have pseudo-diffusive and diffusive mode ( $\gamma_{y}=0$ in the eq. below) for the system obtained from the ansatz (3.15). As before, the dispersion relation reads

$$
\omega\left(k_{y}\right)=-i D_{y} k_{y}^{2}-i \gamma_{y}(T)
$$

In figure 6 we plot these pseudo-diffusive modes of the transverse fluctuations at $T=$ $0.2732 T_{c}$. Again we have the "bubble" behavior, i.e. the meeting of two modes at certain value $k=k_{c}$ and the analysis of the spectrum is analog to that performed for the $a_{y}$ fluctuations in the longitudinal mode. Figure 6 also shows how this critical value $k_{c}$ depends on the temperature. It has a maximum value at the temperature at which the two diffusive modes are the furthest one from the other at zero momentum. Moreover, at very low temperatures and near the critical temperature they are almost at the same place on the imaginary axis. This fact produces a small $k_{c}$ in those regimes. 

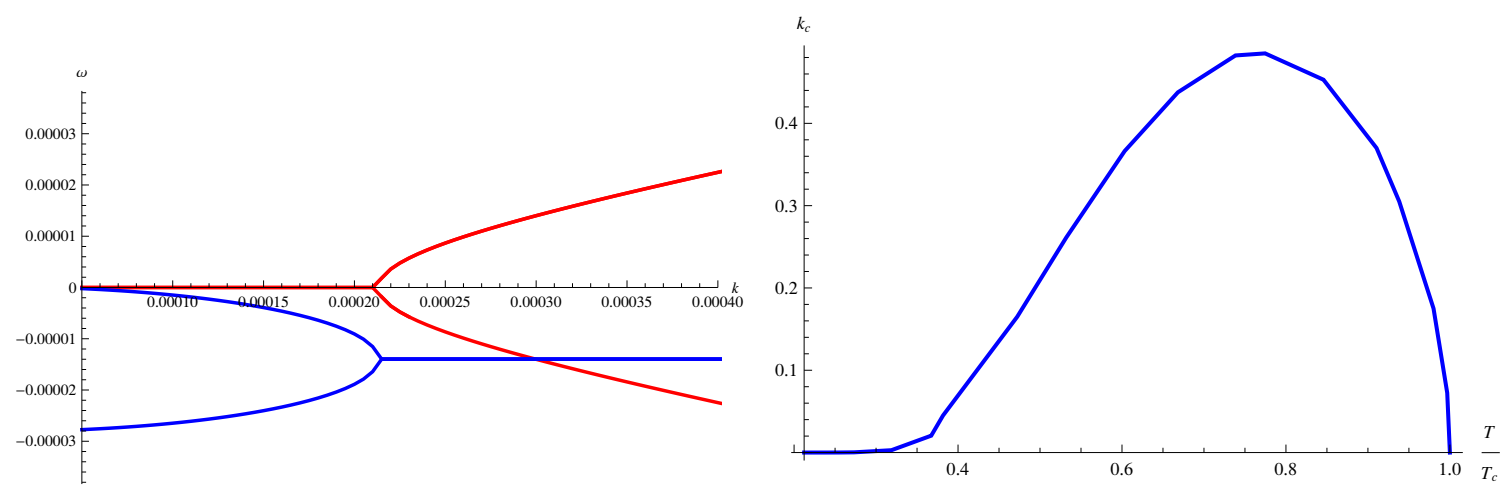

Figure 6. (Left) (Pseudo-)diffusive modes for the transverse sector $I I$ fluctuations at $T=0.2732 T_{c}$. (Right) Critical value of $k$ as a function of the temperature.

\section{Conclusions}

In this paper we look for hydrodynamic modes of a $2+1$ dimensional $p$-wave superfluid. In order to obtain this modes we review its dual gravity background in the probe limit, which is an asymptotically AdS planar charged black hole with a SU(2) gauge field living on it. We work in the non-backreacting limit and we use holography to compute the poles of the current-current retarded Green functions of the dual field theory. Note that in this bottom-up approach we can not say anything about the microscopic theory that originate the superconducting character of the material but we can study its phenomenology. The AdS/CFT dictionary allow us to obtain the poles of the retarded Green function through the computation of the QNM of the gravity dual. Then with this plain in mind we computed the QNM in the geometry dual to the $p$-wave superconductor. We separate the fluctuations of the gauge field in two sectors which we called longitudinal and transverse because they propagate parallel or orthogonal with respect to the direction of the condensate respectively. As expected by the rotational symmetry present in the unbroken phase we have the same number of hydrodynamic modes in both sectors. For the longitudinal modes we find two subsystem of equations, the first one presents one diffusive mode and two sound modes. We computed the velocity of the second sound for these modes and its attenuation as a function of the temperature. We noted that the diffusive mode meets an excited mode at some value of the momentum $k_{c}$ and we made a plot of its temperature dependence. The second subsystem leads to one pseudo-diffusive and one diffusive modes. We observe that the behavior of $k_{c}(T)$ for this modes is different that in the cases where the diffusive modes meet the first excited mode. On the other hand, we obtain the same kind of modes in the transverse sector. One diffusive mode and two sound modes for what was called sector $I$ and one pseudo-diffusive and one diffusive modes for sector II. Again, we plot the second sound velocity as a function of the temperature and observe that it has the same behavior that in the longitudinal case at low temperatures and at $T \sim T_{c}$.

In view of the work [21], the modes found on the present paper can be viewed as responsible of the late time behavior of a $p$-wave superconductor. In particular we noticed a phase transition from a non oscillatory regime to an oscillatory one for inhomogeneous 
quenches with a characteristic wave number greater than $k_{c}$ and followed the behavior of $k_{c}$ as a function of the temperature. As a future work it would be interesting to explicitly check this affirmation by quenching the $p$-wave and computing its late time behaviour. Moreover it could be interesting to see if the hydrodynamic modes that we found could also be obtained using the hydrodynamic equations of [8].

Another possible extension to this work would be to analize the quasinormal modes of the so called $s+p$-wave phase introduced in [38]. This model arises naturally in the low temperature regime of [24] and field theory calculations made in [39] suggest that roton-like exitations may be found.

\section{Acknowledgments}

We would like to thank Daniel "epic" Areán, David Blanco, Richard Davison, Nikolaos Kaplis, Steffen Klug, Guille Silva and Jan Zaanen for useful discussions and comments. We would also like to thank ICTP's Spring School in String Theory and related topics where part of this work was done. R.A. would like to thank to Lorentz Leiden Institute for cordiality during his visit. I.S.L. would like to thank Romi and el Fresco for hospitality during his visit to Bariloche and ICTP where part of this work was done in the context of the STEP program. The work of R.A. was supported by CONICET, CNEA and Universidad Nacional de Cuyo, Argentina.

Open Access. This article is distributed under the terms of the Creative Commons Attribution License (CC-BY 4.0), which permits any use, distribution and reproduction in any medium, provided the original author(s) and source are credited.

\section{References}

[1] J.M. Maldacena, The large- $N$ limit of superconformal field theories and supergravity, Int. J. Theor. Phys. 38 (1999) 1113 [hep-th/9711200] [INSPIRE].

[2] S.S. Gubser, I.R. Klebanov and A.M. Polyakov, Gauge theory correlators from noncritical string theory, Phys. Lett. B 428 (1998) 105 [hep-th/9802109] [INSPIRE].

[3] E. Witten, Anti-de Sitter space and holography, Adv. Theor. Math. Phys. 2 (1998) 253 [hep-th/9802150] [INSPIRE].

[4] S.A. Hartnoll, Lectures on holographic methods for condensed matter physics, Class. Quant. Grav. 26 (2009) 224002 [arXiv:0903.3246] [INSPIRE].

[5] S.A. Hartnoll, Horizons, holography and condensed matter, arXiv:1106.4324 [INSPIRE].

[6] J. McGreevy, Holographic duality with a view toward many-body physics, Adv. High Energy Phys. 2010 (2010) 723105 [arXiv:0909.0518] [INSPIRE].

[7] D. Musso, Introductory notes on holographic superconductors, arXiv:1401.1504 [INSPIRE].

[8] C. Hoyos, B.S. Kim and Y. Oz, Odd Parity Transport In Non-Abelian Superfluids From Symmetry Locking, JHEP 10 (2014) 127 [arXiv:1404.7507] [INSPIRE].

[9] S.S. Gubser and S.S. Pufu, The gravity dual of a p-wave superconductor, JHEP 11 (2008) 033 [arXiv:0805.2960] [INSPIRE]. 
[10] P. Basu, J. He, A. Mukherjee and H.-H. Shieh, Hard-gapped Holographic Superconductors, Phys. Lett. B 689 (2010) 45 [arXiv:0911.4999] [InSPIRE].

[11] R.E. Arias and I.S. Landea, Backreacting p-wave superconductors, JHEP 01 (2013) 157 [arXiv:1210.6823] [INSPIRE].

[12] M. Ammon, J. Erdmenger, V. Grass, P. Kerner and A. O'Bannon, On Holographic p-wave Superfluids with Back-reaction, Phys. Lett. B 686 (2010) 192 [arXiv:0912.3515] [INSPIRE].

[13] P. Kovtun, Lectures on hydrodynamic fluctuations in relativistic theories, J. Phys. A 45 (2012) 473001 [arXiv:1205.5040] [INSPIRE].

[14] D.T. Son and A.O. Starinets, Minkowski space correlators in AdS/CFT correspondence: recipe and applications, JHEP 09 (2002) 042 [hep-th/0205051] [INSPIRE].

[15] H.-P. Nollert, Quasinormal modes: the characteristic 'sound' of black holes and neutron stars. Topical review, Class. Quant. Grav. 16 (1999) R159 [inSPIRE].

[16] E. Berti, V. Cardoso and A.O. Starinets, Quasinormal modes of black holes and black branes, Class. Quant. Grav. 26 (2009) 163001 [arXiv:0905.2975] [INSPIRE].

[17] G. Policastro, D.T. Son and A.O. Starinets, From AdS/CFT correspondence to hydrodynamics, JHEP 09 (2002) 043 [hep-th/0205052] [INSPIRE].

[18] G. Policastro, D.T. Son and A.O. Starinets, From AdS/CFT correspondence to hydrodynamics. 2. Sound waves, JHEP 12 (2002) 054 [hep-th/0210220] [INSPIRE].

[19] C.P. Herzog and S.S. Pufu, The second sound of SU(2), JHEP 04 (2009) 126 [arXiv: 0902.0409] [INSPIRE].

[20] I. Amado, M. Kaminski and K. Landsteiner, Hydrodynamics of holographic superconductors, JHEP 05 (2009) 021 [arXiv: 0903.2209] [INSPIRE].

[21] M.J. Bhaseen, J.P. Gauntlett, B.D. Simons, J. Sonner and T. Wiseman, Holographic Superfluids and the Dynamics of Symmetry Breaking, Phys. Rev. Lett. 110 (2013) 015301 [arXiv:1207.4194] [INSPIRE].

[22] S.A. Hartnoll, C.P. Herzog and G.T. Horowitz, Building a Holographic Superconductor, Phys. Rev. Lett. 101 (2008) 031601 [arXiv:0803.3295] [InSPIRE].

[23] S.A. Hartnoll, C.P. Herzog and G.T. Horowitz, Holographic superconductors, JHEP 12 (2008) 015 [arXiv:0810.1563] [INSPIRE].

[24] I. Amado et al., Holographic Type II Goldstone bosons, JHEP 07 (2013) 108 [arXiv: 1302.5641] [INSPIRE].

[25] R.-G. Cai, L. Li and L.-F. Li, A holographic p-wave superconductor model, JHEP 01 (2014) 032 [arXiv:1309.4877] [INSPIRE].

[26] S. Gangopadhyay and D. Roychowdhury, Analytic study of properties of holographic p-wave superconductors, JHEP 08 (2012) 104 [arXiv:1207.5605] [INSPIRE].

[27] I. Amado et al., Holographic Superfluids and the Landau Criterion, JHEP 02 (2014) 063 [arXiv: 1307.8100] [INSPIRE].

[28] N. Jokela, G. Lifschytz and M. Lippert, Flowing holographic anyonic superfluid, JHEP 1410 (2014) 21 [arXiv:1407.3794] [INSPIRE]. 
[29] A.S. Miranda, J. Morgan and V.T. Zanchin, Quasinormal modes of plane-symmetric black holes according to the AdS/CFT correspondence, JHEP 11 (2008) 030 [arXiv:0809.0297] [INSPIRE].

[30] K.R. Atkins and R.A. Stasior, First sound in liquid helium at high pressures, Can. J. Phys. 31 (1953) 1156.

[31] V.P. Peshkov, Second Sound in Helium II, Sov. Phys. JETP 11 (1960) 580.

[32] E. Taylor, H. Hu, X.-J. Liu, L.P. Pitaevskii, A. Griffin and S. Stringari, First and second sound in a strongly interacting Fermi gas, Phys. Rev. A 80 (2009) 053601 [arXiv: 0905. 0257].

[33] A. Buchel and C. Pagnutti, Bulk viscosity of $N=2^{*}$ plasma, Nucl. Phys. B 816 (2009) 62 [arXiv: 0812.3623] [INSPIRE].

[34] O. Bergman, N. Jokela, G. Lifschytz and M. Lippert, Striped instability of a holographic Fermi-like liquid, JHEP 10 (2011) 034 [arXiv:1106.3883] [INSPIRE].

[35] R.A. Davison and A.O. Starinets, Holographic zero sound at finite temperature, Phys. Rev. D 85 (2012) 026004 [arXiv:1109.6343] [InSPIRE].

[36] N. Jokela, G. Lifschytz and M. Lippert, Magnetic effects in a holographic Fermi-like liquid, JHEP 05 (2012) 105 [arXiv: 1204.3914] [INSPIRE].

[37] B.S. DiNunno, M. Ihl, N. Jokela and J.F. Pedraza, Holographic zero sound at finite temperature in the Sakai-Sugimoto model, JHEP 04 (2014) 149 [arXiv:1403.1827] [INSPIRE].

[38] I. Amado, D. Arean, A. Jimenez-Alba, L. Melgar and I. Salazar Landea, Holographic $s+p$ Superconductors, Phys. Rev. D 89 (2014) 026009 [arXiv: 1309.5086] [INSPIRE].

[39] V.P. Gusynin, V.A. Miransky and I.A. Shovkovy, Spontaneous rotational symmetry breaking and roton-like excitations in gauged $\sigma$-model at finite density, Phys. Lett. B 581 (2004) 82 [hep-ph/0311025] [INSPIRE]. 